\title{
INCLUSIÓN, ADSCRIPCIÓN E IDENTIDAD ÉTNICA EN ESTUDIANTES DE LA UNIVERSIDAD DE TARAPACÁ, FRONTERA NORTE DE CHILE*
}

\author{
INCLUSION, ASCRIPTION AND ETHNIC IDENTITY IN STUDENTS \\ OF UNIVERSIDAD DE TARAPACÁ, NORTHERN BORDER OF CHILE
}

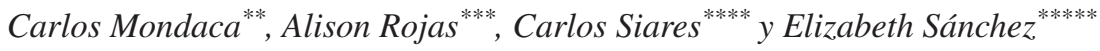

\begin{abstract}
El objetivo de este artículo es establecer una línea base que identifica y caracteriza la distribución de las adscripciones étnicas de los estudiantes de pregrado de la Universidad de Tarapacá (Sede Arica e Iquique). Metodológicamente por medio de una encuesta estructurada aplicada al universo total del alumnado se obtuvo 82,02\% de participación, lo que permite tener una aproximación acertada de las adscripciones étnicas de los estudiantes y su relación con la proveniencia de tipo educacional, certificación indígena y participación en asociaciones y agrupaciones. Con este análisis preliminar podemos sostener la importancia de manejar información acerca de adscripciones étnicas de los estudiantes para promover una política institucional en materia de inclusión de la variable indígena al momento de elaborar el rediseño curricular y el plan de gestión estratégico de la universidad desde una perspectiva con pertinencia cultural concreta, más que solo una declaración de principios.
\end{abstract}

Palabras claves: Arica y Parinacota, Norte de Chile, adscripciones étnicas, Universidad de Tarapacá.

The aim of this article is to establish a baseline that identifies and characterizes the distribution of ethnic affiliations of undergraduate students at the University of Tarapacá (Arica and Iquique Headquarters). Methodologically, through a structured survey applied to the total universe of students, $82.02 \%$ of participation was obtained, which allows a correct approximation of the students' ethnic affiliations and their relation with the origin of educational type, indigenous certification and Participation in associations and groups. With this preliminary analysis, we can sustain the importance of managing information about students' ethnic affiliations in order to promote an institutional policy regarding the inclusion of the indigenous variable at the time of elaborating the curricular redesign and the strategic management plan of the university from a perspective with Concrete cultural relevance, rather than just a declaration of principles.

Key words: Arica and Parinacota, Northern Chile, ethnic ascriptions, University of Tarapacá.

\section{Introducción}

La política pública en materia de Interculturalidad puesta en la agenda internacional desde 1990 en Jomtiem por la $\mathrm{UNESCO}^{1}$, irrumpe con fuerza la necesidad de contribuir en el ámbito de la educación desde una mirada de igualdad de oportunidades para todos los sectores de la sociedad ${ }^{2}$. Lo que se traduce que el 2014, en el proceso de seguimiento de la implementación de esta política en todos los países, se informa que pese a no haberse cumplido todos los acuerdos tomados en reuniones anteriores, existe un avance significativo en algunos aspectos del programa de Educación para Todos, lo que se traduce que el 2015 se publique la Declaración de
Incheon, titulada: "Educación 2030: Hacia una educación inclusiva y equitativa de calidad y un aprendizaje a lo largo de la vida para todos" ${ }^{\text {"3. Desde }}$ entonces, los sistemas educativos mundiales han comenzado a reestructurar sus políticas públicas en temáticas de inclusión, y de forma particular las Instituciones de Educación Superior (IES), sobre todo aquellas que tienen bajo su responsabilidad la Formación Inicial Docente (FID).

En el caso del Estado chileno, antes de estos foros, surgen iniciativas desde los gobiernos de turno (1995), que comenzaron a instalar programas de Educación Intercultural Bilingüe del Ministerio de Educación (MINEDUC) y de la Corporación Nacional de Desarrollo Indígena (CONADI) en

\footnotetext{
* Resultado Proyecto Fondecyt No 1160976 y Proyecto de Investigación en Educación UTA, Código 5712-15. Se agradece el apoyo del Convenio de Desempeño Regional UTA-MINEDUC 1401.

** Universidad de Tarapacá, Departamento de Educación. Arica, Chile. Correo electrónico: cemondacar@uta.cl

*** Universidad de Tarapacá, Departamento de Docencia de Pregrado. Arica, Chile. Correo electrónico: arojasm@uta.cl

*****Universidad de Tarapacá, Departamento de Desarrollo Tecnológico. Arica, Chile. Correo electrónico: csiares@uta.cl

****** Universidad de Tarapacá, Convenio Desempeño Regional. Arica, Chile. Correo electrónico: esanchezg@uta.cl
} 
áreas con población indígena, los que han ido escalando lentamente desde el área rural a la urbana, incorporando en el último tiempo el sector de lengua indígena como parte del currículum escolar en las escuelas. En la última década el tema de la inclusión ha tomado relevancia producto de hechos de discriminación y violencia ocurridos en el país.

Desde entonces, el MINEDUC ha centrado su preocupación en temas de inclusión relacionados con la convivencia escolar, el 2013 se publicó el texto: Discriminación en el contexto escolar. Orientaciones para promover una escuela inclusiva, donde se compromete a toda la comunidad educativa a trabajar en pos de la inclusión mediante políticas de acción afirmativa o de discriminación positiva. Todo este trabajo se consolida con la promulgación en mayo de 2015 de la Ley de Inclusión ( $\left.\mathrm{N}^{\circ} 20.845\right)$, que tiene como inicio de vigencia marzo de 2016, la que toma en cuenta las disposiciones del Informe de Seguimiento del Programa "Educación para Todos" 4 . Desde este contexto político, surgen desafíos importantes en materia de educación universitaria, pues las IES, junto con sus procesos de acreditación, incorporan en sus planes estratégicos acciones concretas de inclusión e interculturalidad ${ }^{5}$.

De acuerdo con estos objetivos y metas trazadas a nivel mundial, nuestra región con una larga historia multiétnica y multicultural como parte de una sociedad fronteriza, posee una universidad pública y estatal donde interactúan y se articulan una diversidad de identidades (nacionales, étnicas, género, sociopolíticas, entre otras); más allá de las fronteras geopolíticas, esta situación se debe tomar en cuenta en sus procesos de formación inicial de profesionales como parte de un territorio con diversidad cultural.

De acuerdo con lo anterior, el propósito de esta investigación es proveer de información para generar una línea base respecto de las adscripciones étnicas de los estudiantes de pregrado de la Universidad de Tarapacá para la gestión eficiente de una política institucional en materia de inclusión de la variable indígena al momento de elaborar el rediseño curricular y el plan de gestión estratégico de la universidad desde una perspectiva con pertinencia cultural concreta, más que solo una declaración de principios. Para esto es indispensable contar con antecedentes estadísticos como parte de su quehacer funcional, tanto a nivel académico y de investigación como de gestión y vinculación con el medio externo.

\section{Arica y Parinacota la región más intercultural del norte de Chile}

La Región de Arica y Parinacota se fue constituyendo demográficamente con el tiempo por la presencia de diferentes colectivos humanos, no es menor que en la prehistoria regional se constituya como un territorio multiétnico con la ocupación de distintos grupos humanos desde el borde costero hasta el gélido altiplano regional. En este contexto, de acuerdo con estudios etnohistóricos apoyados por los resultados de estudios arqueológicos regionales, se produjo una compleja red de relaciones étnicas diferenciadas por pisos agroecológicos que durante fases muestran un panorama de procesos de ocupación de culturas como la Tiwanacu e Inca y desarrollos regionales.

Con la llegada de los colonizadores en el siglo XVI y la mestización producida por la aparición de diversas castas asociadas a la pigmentocracia, la región presenta un tejido de mixturas étnicas que se manifiestan en una hibridación cultural que fue configurando un escenario transcultural ${ }^{6}$. Hispanos, criollos, mestizos, indios y población afro fueron la amalgama de diversidad presente en la región que sumaron durante los procesos de instauración de las repúblicas y Estados nacionales una inmigración interna entre Chile, Perú y Bolivia y desplazamientos de colectivos regionales del área rural a la urbana. También, los procesos de industrialización y comercio del guano en un primer momento y la articulación con el espacio salitrero trajo consigo una creciente inmigración extranjera. Inmigrantes europeos (croatas, italianos, franceses, españoles, griegos, ingleses, alemanes) y orientales (chinos y japoneses) fueron los que paulatinamente se mezclaron con los locales y se asentaron definitivamente en Arica y Parinacota, siendo un aporte clave para el desarrollo de la región por las diversas actividades económicas que desempeñaban en la ciudad.

Desde la guerra del Pacífico la diversidad de nacionalidades y colectivos étnicos estuvo en conflicto por adscripciones ciudadanas debido al conflicto diplomático con Perú, lo que se tradujo en la venida de oleadas de inmigrantes del norte chico y zona central de Chile, por el supuesto plebiscito que debería definir la soberanía de estos territorios que estuvieron en statu quo casi cincuenta años, durante el llamado proceso de chilenización que tuvo una solución con el Tratado de Lima en 1929, esto 
significó la salida de muchos coterráneos fundamentalmente población de afrodescendientes al Perú.

El siguiente proceso de inflexión importante en materia de diversidad se configuró a partir de la creación del Puerto Libre y de la Junta de Adelanto, que generó un polo de desarrollo económico en la zona, transformando a la región en un nodo de atracción de población nacional y local, destacándose la migración translocal de la población originaria del interior de Arica. Este fenómeno provocó el aumento del crecimiento demográfico y económico de la región con una diversidad cultural que la componen un conjunto de identidades locales, a las que hay que sumar la constante y permanente migración transfronteriza de grupos de peruanos y bolivianos que se ha incrementado en los últimos 30 años y la reciente migración de colectivos transnacionales colombianos y ecuatorianos. Lo que dificulta aún más la diversidad presente en nuestro territorio. Todo este panorama actual se configuraría como un fenómeno producto de la globalización, ya que estos movimientos migratorios irrumpen con nuevas formas de movilidad social y de inserción en nuestra región.

Actualmente el territorio que comprende a la actual región de Arica y Parinacota según los datos de la encuesta CASEN 2013, desde el punto de vista étnico existen 54.075 (31,5\%) personas de la población que declara pertenecer a una etnia, siendo aymaras el $88,1 \%$, mapuches $6,9 \%$, y otros $5 \%$. Asimismo, de acuerdo con los resultados del primer estudio realizado el 2013 por el INE, donde se aplicó la encuesta de caracterización de la población afrodescendiente de la Región de Arica y Parinacota, se puede establecer una población estimada de 8.415 , correspondiente a $4,7 \%$ del total de la población total de la región.

También dentro de este total se debe considerar a 8.018 inmigrantes de distintas nacionalidades (CASEN, 2013) que se han asentado en la zona, correspondiendo al 2,1\% de la población regional. Esta situación es especialmente significativa en nuestra región, donde, utilizando los datos del Censo 2012 solo como fuente de comparación referencial (ya que la migración se ha intensificado en los últimos años), los extranjeros representarían el 5,8\% de la población total (12.299 personas), donde los peruanos constituirían 39,6\% (4.821 personas) del total de migrantes, superados solo por los residentes bolivianos que agrupan $48 \%$ de la población migrante regional (5.903 personas).

\section{La Universidad del Estado en la frontera norte: La Universidad de Tarapacá}

Un estudio del Centro Interuniversitario de Desarrollo del 2010 (CINDA) sostiene la importancia de la implantación de programas de acción afirmativa en las universidades chilenas, debido a que las políticas públicas debían tomar en cuenta que:
"En la formación profesional y de líderes debe estar representada la composición étnica de la población.
La universidad, como centro principal de formación de profesionales líderes, debe graduar generaciones de acuerdo a esa representación de la población.
Para graduar esas generaciones es necesario admitir cohortes de estudiantes étnicamente representativas".

\section{Concluían que}
“...la diversidad étnica y cultural no es un contribuyente incidental a una misión universitaria, sino que deberían ser parte de la misión de la universidad, así como lo son la responsabilidad social, la investigación y la extensión relevantes para el desarrollo local y nacional" (Alvarado, et al., 2010)

En este sentido, la Universidad de Tarapacá como Institución de Educación Superior de carácter estatal, con una ubicación en la frontera norte de Chile en su último Plan Estratégico de Desarrollo Institucional (2011-2016) se planteó mejorar la calidad en la formación de las carreras que impartía (Proceso de Acreditación 2012), para esto debía asumir los desafíos que la diversidad y desigualdad socioeconómica y sociocultural del estudiantado les presentaba.

Por lo anterior, en el Modelo Educativo Institucional (2012:20) se planteó de manera oficial que:

"La Universidad de Tarapacá manifiesta su compromiso permanente con el desarrollo de valores de inclusión, respeto y promoción de la diversidad cultural y la interculturalidad. En este sentido, su compromiso es con el desarrollo de potencialidades de personas y 
grupos de diferentes culturas, construyendo puentes de relación que enriquezcan a toda la comunidad".

Esto no quiere decir que antes de esta declaración de principios no se hayan implementado iniciativas frente al tema de inclusión de grupos étnicos; de forma general, en las últimas décadas se han desarrollado varias iniciativas e intervenciones relacionadas con becas y ayudas externas (principalmente de la CONADI) a los estudiantes indígenas que contemplan recursos y residencia estudiantil, entre otros ${ }^{7}$.

Desde el punto de vista de la formación docente, se han desarrollado algunas iniciativas en cursos, talleres y electivos de formación general de lengua, cultura y patrimonio aymara, que son el resultado de proyectos de unidades académicas que se logran con recursos internos y externos. Muy por el contrario, la investigación científica de la universidad está orientada a desarrollar líneas de investigación específicas respecto del tema étnico en el área de la antropología, arqueología, psicología, historia y geografía, entre otras. Conocimiento que se difunde principalmente a pares en congresos y publicaciones en revistas científicas ${ }^{8}$.

En el contexto curricular, los procesos de autoevaluación y acreditación de las carreras han llevado a implementar en algunas carreras la variable de diversidad incorporando asignaturas desde una perspectiva intercultural: Pedagogía Básica, Educación Parvularia, Enfermería, Pedagogía en Lenguaje y Comunicación y Antropología, lo que se manifiesta en una política no instalada institucionalmente desde el ámbito del diseño curricular para el conjunto de las carreras $(48)^{9}$.

En todo este proceso uno de los principales problemas de definición de qué entendemos como población indígena y la principal dificultad es saber a ciencia cierta cuántos son en nuestra casa de estudios. Diversos han sido los criterios utilizados, y los indicadores van desde contabilizar estudiantes con apellidos indígenas, con beca indígena, con certificación de la CONADI, con certificados emanados de la comunidad, asociaciones y organizaciones indígenas, como certificados de residencia de pueblos históricamente señalados como indígenas.

Y este problema no es fácil de zanjar, pues en la bibliografía nacional se ha instalado la problemática de cómo definiremos las adscripciones étnicas desde hace un buen tiempo, pues tal como lo señalan
Gundermann y Vergara (2009), la situación de la conformación de las comunidades y organizaciones andinas en el norte de Chile ha sido bastante compleja, aunque sostienen que la comunidad andina pese a los cambios ocurridos en ella, mantiene vigencia desde una perspectiva translocal, que pese a la dinámica de transformaciones aún es importante el parentesco y sentido de pertenencia a un territorio a la hora de estimar que es ser indígena en Chile.

También se utilizaron en este trabajo los criterios metodológicos de Oyarce, Pedrero y Pérez (2005), para clarificar la identificación y cuantificación de las adscripciones étnicas de los estudiantes de la Universidad de Tarapacá y las consideraciones de Gavilán y Lagos (2014) acerca de los antecedentes relacionados con el proceso de acreditación de la condición indígena con la CONADI, como un criterio válido de utilizar a la hora de contabilizar poblaciones de estudiantes de pueblos originarios, teniendo como eje central el parentesco.

\section{Metodología}

De acuerdo con lo anterior, uno de los primeros problemas metodológicos fue determinar los criterios para definir cómo mediríamos la adscripción étnica para diseñar el instrumento y sus correspondientes preguntas de acuerdo con indicadores que permitieran tener una mejor estadística de los estudiantes indígenas. Resultó relevante el trabajo de Oyarce, Pedrero y Pérez (2005: 12), pues tomamos en cuenta sus criterios de singularidad étnica para hacer preguntas por grupo indígena: siendo relevantes la autoidentificación $(61,4 \%)$, parentesco $(22,9 \%)$, lo que asociamos a su reconocimiento vía certificación estatal, y el resto de marcadores con menor importancia porcentual a la hora de hacer esta pregunta las relacionamos con preguntas más amplias a su asociación con la mantención de rasgos culturales $(19,3 \%)$, apellidos $(9,8 \%)$ y territorio $(8,4 \%)$, el indicador de rasgos físicos $(3,8 \%)$ no lo tomamos en consideración en la encuesta aplicada; en otros $(2,4 \%)$, preguntamos por ciudad de origen, colegio de estudio, beneficios y becas estudiantiles.

La metodología utilizada es de tipo mixta, pues estadísticamente es cuantitativa, pero en el cruce de variables logramos determinar análisis cualitativos. Se utilizó una encuesta estructurada con opciones múltiples, la que incluyó las siguientes preguntas: datos generales (sede de estudio, carrera, colegio en que desarrolló la enseñanza media); su adscripción 
étnica; certificación CONADI; participación en asociaciones indígenas y tipos de beneficios con los que cuentan al interior de la Universidad.

\subsection{Procedimiento}

La aplicación de la encuesta se llevó a cabo entre abril y noviembre del 2015. Para abarcar la mayor cantidad de estudiantes de pregrado, la encuesta fue incorporada en el acceso a los servicios que entrega la Intranet de la institución a sus alumnos, para ello se utilizó una plataforma web diseñada con leguaje PHP y los datos fueron almacenados en las estructuras de la base de datos implementada en ORACLE. Los participantes respondieron voluntariamente. Una vez aplicados los instrumentos, se procedió a estructurar las respuestas y eliminar los datos que no cumplían con las condiciones necesarias para el presente estudio, como por ejemplo, respuestas incompletas en las escalas.

\subsection{Análisis de datos}

Se efectuó un análisis descriptivo para obtener las frecuencias (porcentajes) de las respuestas de cada una de las preguntas incluidas en la encuesta. Este análisis fue llevado a cabo mediante el programa Statistical Package Social Science (SPSS) versión 22 y se generaron descriptivos generales de los resultados obtenidos en la encuesta.

\section{Resultados}

\subsection{Datos descriptivos}

De 8.547 alumnos matriculados al 2015 en la Universidad de Tarapacá, 82,02\% (7.011) respondió a la encuesta aplicada. De ellos 82,5\% pertenece a la sede de Arica (5.785), 17,4\% a la sede de Iquique (1.220). Es importante señalar que el mayor porcentaje de ellos provenía de un establecimiento educacional particular subvencionado $(56,4 \%)$, le siguen en segundo lugar y tercer lugar respectivamente los colegios municipalizados $(36,9 \%)$ y los particulares privados $(3,8 \%)$. Finalmente, un porcentaje menor $(2,9 \%)$ no identificó el establecimiento en que efectuó su enseñanza media o básica.

\subsection{Tablas de contingencia}

En primer lugar, mostraremos una tabla de contingencia que resume la sede de estudio y el estadístico de autoidentificación sobre si: ¿Se siente perteneciente a algún pueblo originario? Sobre la base de los que respondieron sí o no y su porcentaje de representatividad frente al total de alumnos por sede y el total de ambas sedes (Tabla 1).

Como podemos observar el porcentaje de respuesta resulta bastante significativo con relación al universo total por sede, ya que para ambas es por sobre el $80 \%$ del universo total de estudiantes de la Universidad de Tarapacá.

Respecto de los estudiantes que se autoidentifican con la opción Sí, en la Sede de Arica llegan a $39,06 \%$ de la muestra y $32,03 \%$ del total de la matrícula, lo que representa que un tercio de la población estudiantil de la Universidad de Tarapacá se autoadscribe con un pueblo originario.

En el caso de la sede Iquique, se llega a 35,25\% de la muestra y $21,3 \%$ del total de la matrícula, manifestando, al igual que la cifras de Arica, un importante número de alumnos con autoidentificación con un pueblo originario. De este total por sede, en la pregunta a ¿A cuál de los pueblos originarios se siente parte?, tenemos de la muestra desagregada por pueblos originarios los siguientes resultados (Tabla 2).

Tabla 1. Autoidentificación étnica en la Universidad de Tarapacá.

\begin{tabular}{|c|c|c|c|c|c|}
\hline \multirow{2}{*}{ Sede } & \multicolumn{2}{|c|}{$\begin{array}{c}\text { ¿Se siente perteneciente a algún } \\
\text { pueblo originario? }\end{array}$} & \multirow{2}{*}{ Total muestra } & \multirow{2}{*}{$\begin{array}{l}\% \text { del total } \\
\text { matrícula }\end{array}$} & \multirow{2}{*}{$\begin{array}{l}\text { Total matrícula UTA } \\
\text { a abril de } 2015\end{array}$} \\
\hline & Sí & No & & & \\
\hline Arica & 2.260 & 3.525 & 5.785 & 81,99 & 7.055 \\
\hline Iquique & 318 & 902 & 1.220 & 81,76 & 1.492 \\
\hline Total & 2.579 & 4.432 & 7.011 & 82,02 & 8.547 \\
\hline
\end{tabular}


Tabla 2. Autoidentificación étnica en la Universidad de Tarapacá.

\begin{tabular}{|c|c|c|c|c|}
\hline \multirow{2}{*}{ Pueblo originario } & \multicolumn{4}{|c|}{ Sede en la que estudia } \\
\hline & Arica & Iquique & Sede no identificada & Total \\
\hline \multirow{2}{*}{ Atacameño } & 67 & 11 & $\mathbf{0}$ & 78 \\
\hline & $1.0 \%$ & $0,2 \%$ & $0,0 \%$ & $1,1 \%$ \\
\hline \multirow{2}{*}{ Quechua } & 49 & 14 & $\mathbf{0}$ & 63 \\
\hline & $0,7 \%$ & $0,2 \%$ & $0,0 \%$ & $0,9 \%$ \\
\hline \multirow{2}{*}{ Aymara } & 1.640 & 189 & 0 & 1829 \\
\hline & $23,4 \%$ & $2,7 \%$ & $0,0 \%$ & $26,1 \%$ \\
\hline \multirow{2}{*}{ Kolla } & 13 & 5 & 0 & 18 \\
\hline & $0,2 \%$ & $0,1 \%$ & $0,0 \%$ & $0,3 \%$ \\
\hline \multirow{2}{*}{ Diaguita } & 70 & 22 & $\mathbf{0}$ & 92 \\
\hline & $1,0 \%$ & $0,3 \%$ & $0,0 \%$ & $1,3 \%$ \\
\hline \multirow{2}{*}{ Rapa Nui } & 7 & 5 & 0 & 12 \\
\hline & $0,1 \%$ & $0,1 \%$ & $0,0 \%$ & $0,2 \%$ \\
\hline \multirow{2}{*}{ Mapuche } & 214 & 55 & $\mathbf{0}$ & 269 \\
\hline & $3,1 \%$ & $0,8 \%$ & $0,0 \%$ & $3,8 \%$ \\
\hline \multirow{2}{*}{ Kawésqar } & 3 & 0 & 0 & 3 \\
\hline & $0,0 \%$ & $0,0 \%$ & $0,0 \%$ & $0,0 \%$ \\
\hline \multirow{2}{*}{ Afrodescendientes } & 144 & 3 & 1 & 148 \\
\hline & $2,1 \%$ & $0,0 \%$ & $0,0 \%$ & $2,1 \%$ \\
\hline \multirow{2}{*}{ Otros } & 53 & 14 & 0 & 67 \\
\hline & $0,8 \%$ & $0,2 \%$ & $0,0 \%$ & $1,0 \%$ \\
\hline \multirow{2}{*}{ A ninguno } & 3.525 & 902 & 5 & 4432 \\
\hline & $50,3 \%$ & $12,9 \%$ & $0,1 \%$ & $63,2 \%$ \\
\hline
\end{tabular}

En la Sede de Arica, así como la sede de Iquique, la autoadscripción con un pueblo originario releva al pueblo aymara en primer lugar (26\%), información que se condice completamente con la historia del país, porque este pueblo habita en la zona norte y en mayor proporción en la Región de Arica y Parinacota y Región de Tarapacá.

En segundo lugar, respecto de la autoadscripción con un pueblo originario, en ambas sedes se distingue la población mapuche $(3,8 \%)$, dato que si bien se encuentra en una proporción menor y lejana en comparación al primer lugar, resalta, ya que a diferencia de los aymaras, este pueblo habita en el sur de Chile, no obstante ello, se debe considerar que la Universidad de Tarapacá, al ser estatal, recibe ingresos a nivel nacional.

El estudio desagregado por Carreras arrojó los siguientes resultados (Tabla 3 ):

En cuanto al detalle por Facultad-Escuela/ carrera, es posible evidenciar que un tercio de los estudiantes que se autoidentifican con un pueblo originario pertenecen a la Facultad de Ciencias de la Salud, donde la proporción mayor se ubica en la carrera de Enfermería (10,5\%). Le sigue, las Escuelas de Ingeniería, siendo Ingeniería Civil Industrial la carrera preferente $(6,6 \%)$. Finalmente, la Facultad de Ciencias Sociales y Jurídicas se encuentra en tercer lugar (23\%), donde la proporción mayor se ubica en la carrera de Psicología (10,3\%).

De acuerdo con los datos expuestos, las carreras del área de la salud e Ingenierías son mayormente preferidas por los estudiantes con autoidentificación a un pueblo indígena. Por otra parte, las carreras relacionadas con la pedagogía son las menos elegidas por los estudiantes que manifiestan sentirse perteneciente a un pueblo indígena.

La encuesta recaba información respecto de cuántos de los estudiantes poseían certificación de la CONADI, proporcionándonos el siguiente cuadro (Tabla 4).

Del total de la muestra (Sede Arica e Iquique), es relevante observar que 1.831 estudiantes se manifestaron por la opción Sí, y 5.180 por la opción No. Respecto de aquellos que manifiestan poseer certificación CONADI, 1.713 estudiantes (94\%) se autoidentifica con algún pueblo originario y $6 \%$ declaró inicialmente no sentirse perteneciente a alguno de ellos. Considerando la cifra de estudiantes que se autoadscribe con un pueblo originario y posee certificación CONADI (1.713), es posible mencionar que representan a $24 \%$ del total de la muestra y a $20 \%$ del total de 
Tabla 3. Autoidentificación por Facultad y Carreras de la Universidad de Tarapacá.

\begin{tabular}{|c|c|c|c|c|c|}
\hline Carreras & Sí & No & $\mathrm{N}^{\circ}$ & $\%$ & $\begin{array}{c}\text { Matrícula a } \\
\text { diciembre de } 2015\end{array}$ \\
\hline Enfermería & 228 & 505 & 733 & 10,5 & 901 \\
\hline Arica & 184 & 344 & 528 & & 661 \\
\hline Iquique & 44 & 161 & 205 & & 240 \\
\hline Kinesiología y Rehabilitación & 107 & 214 & 321 & 4,6 & 381 \\
\hline Arica & 98 & 182 & 280 & & 332 \\
\hline Iquique & 9 & 32 & 41 & & 49 \\
\hline Tecnología Médica, Lab. Clínico, Hemat. y Bco. de Sangre & 65 & 94 & 159 & 2,3 & 199 \\
\hline Tecnología Médica, Imagenología y Rayos & 117 & 147 & 264 & 3,8 & 311 \\
\hline Tecnología Médica, Oftalmología y Optometría & 45 & 75 & 120 & 1,7 & 142 \\
\hline Nutrición y Dietética & 94 & 183 & 277 & 4,0 & 353 \\
\hline Arica & 80 & 114 & 194 & & 252 \\
\hline Iquique & 14 & 69 & 83 & & 101 \\
\hline Obstetricia y Puericultura & 104 & 151 & 255 & 3,6 & 299 \\
\hline Psicología & 219 & 503 & 722 & 10,3 & 850 \\
\hline Arica & 145 & 309 & 454 & & 524 \\
\hline Iquique & 74 & 194 & 268 & & 326 \\
\hline Derecho & 166 & 343 & 509 & 7,3 & 821 \\
\hline Arica & 112 & 215 & 327 & & 537 \\
\hline Iquique & 54 & 128 & 182 & & 284 \\
\hline Trabajo Social & 111 & 166 & 277 & 4,0 & 316 \\
\hline Arica & 83 & 112 & 195 & & 220 \\
\hline Iquique & 28 & 54 & 82 & & 96 \\
\hline Antropología & 40 & 82 & 122 & 1,7 & 170 \\
\hline Agronomía & 80 & 108 & 188 & 2,7 & 219 \\
\hline Ingeniería Plan Común & 25 & 25 & 50 & 0,7 & 62 \\
\hline Ingeniería Mecánica/Mecatrónica & 198 & 226 & 424 & 6,0 & 340 \\
\hline Ingeniería Civil Industrial & 172 & 290 & 462 & 6,6 & 566 \\
\hline Arica & 144 & 231 & 375 & & 467 \\
\hline Iquique & 28 & 57 & 85 & & 99 \\
\hline Ingeniería Civil en Computación e Informática & 68 & 119 & 187 & 2,7 & 240 \\
\hline Arica & 55 & 76 & 131 & & 171 \\
\hline Iquique & 13 & 43 & 56 & & 69 \\
\hline Ingeniería Comercial & 109 & 224 & 333 & 4,7 & 299 \\
\hline Ing. en Sistemas de Información y Control de Gestión & 68 & 51 & 119 & 1,7 & 106 \\
\hline Ingeniería Eléctrica/Electrónica & 104 & 140 & 244 & 3,5 & 377 \\
\hline Arica & 89 & 98 & 187 & & 310 \\
\hline Iquique & 15 & 42 & 57 & & 67 \\
\hline Pedagogía en Biología y Cs. Naturales & 6 & 12 & 18 & 0,3 & 24 \\
\hline Contador Auditor (Vespertino) & 38 & 35 & 73 & 1,0 & 102 \\
\hline Diseño Multimedia & 26 & 57 & 83 & 1,2 & 121 \\
\hline Pedagogía en Educación Básica & 52 & 79 & 131 & 1,9 & 153 \\
\hline Arica & 38 & 43 & 81 & & 91 \\
\hline Iquique & 14 & 36 & 50 & & 62 \\
\hline Profesor de Educación Física & 69 & 100 & 169 & 2,4 & 210 \\
\hline Pedagogía en Historia y Geografía & 31 & 92 & 123 & 1,8 & 145 \\
\hline Ingeniería Química Ambiental & 26 & 69 & 95 & 1,4 & 114 \\
\hline Pedagogía Licenciatura en Inglés & 42 & 130 & 172 & 2,5 & 210 \\
\hline Pedagogía en Matemática & 23 & 29 & 52 & 0,7 & 58 \\
\hline Pedagogía en Educación Musical & 3 & 3 & 6 & 0,1 & 9 \\
\hline Educación Parvularia & 76 & 93 & 169 & 2,4 & 205 \\
\hline Arica & 63 & 63 & 131 & & 153 \\
\hline Iquique & 8 & 30 & 38 & & 52 \\
\hline Pedagogía Química y Cs. Naturales & 1 & 1 & 2 & 0,0 & 3 \\
\hline Químico Laboratorista & 29 & 26 & 55 & $0,8 \%$ & 52 \\
\hline Pedagogía en Castellano y Comunicación & 37 & 60 & 97 & 1,4 & 115 \\
\hline Total & 2.579 & 4.432 & 7.011 & $100 \%$ & $* 8.678$ \\
\hline
\end{tabular}

*La matrícula varía entre 150 a 200 estudiantes entre el primer y segundo semestre. 
Tabla 4. Certificación CONADI.

\begin{tabular}{lccc}
\hline \multirow{2}{*}{ ¿Se siente perteneciente a un pueblo originario? } & \multirow{2}{*}{ ¿Posee certificación CONADI? } & \multirow{2}{*}{ Total } \\
\cline { 2 - 3 } & Sí & 866 & 2.579 \\
\hline Se siente perteneciente a un pueblo originario & 1.713 & 4.314 & 4.432 \\
No se siente perteneciente a un pueblo originario & 118 & 5.180 & 7.011 \\
Total & 1.831 & & \\
\hline
\end{tabular}

Tabla 5. Reconocimiento ante la CONADI.

\begin{tabular}{lrrr}
\hline \multirow{2}{*}{ Motivo por el que no se ha reconocido como indígena } & ¿Se siente perteneciente a un pueblo originario? & \multirow{2}{*}{ Total } \\
\cline { 2 - 3 } & Sí & 489 & 838 \\
\hline Desconocimiento & 349 & 95 & 180 \\
Falta de interés & 85 & 14 & 63 \\
No cree en la institucionalidad & 49 & 7 & 17 \\
Para no ser discriminado & 10 & 197 & 448 \\
Otro motivo & 251 & 3.512 & 3.634 \\
Omite respuesta & 122 & 118 & 1.831 \\
Sí estoy reconocido & 1.713 & & \\
\hline
\end{tabular}

la matrícula. Lo anterior evidencia un porcentaje importante de autoidentificación y adscripción étnica en los estudiantes de la Universidad de Tarapacá, desde el punto de vista legal. A partir de los datos expuestos es posible relevar que existen 118 estudiantes que pese a estar acreditados por pertenecer a una de las etnias reconocidas del país (Certificación CONADI), manifiestan no sentirse parte de un pueblo originario, dato que da cuenta de una cifra menor que aún no se reconoce como descendiente de indígenas. En cuanto al motivo por el que aquellos que sí se sienten parte de un pueblo originario no se han reconocido como tal ante la CONADI, el motivo que prima es el desconocimiento, le sigue "otro motivo" y en tercer lugar la falta de interés (Tabla 5). Estos datos son de suma utilidad, ya que permiten generar acciones para aumentar la cifra de personas reconocidas y, a su vez, los datos de los Censos y estudios asociados a los pueblos indígenas reflejarían con mayor exactitud la realidad de la región.

Se consideró relevante consultar por la participación de los estudiantes en asociaciones u organizaciones indígenas, pudiéndose evidenciar que del total de alumnos que declara sentirse parte de un pueblo originario solo el $40 \%$ participa en alguna asociación u organización. En esta misma línea, resulta relevante que 105 estudiantes que manifiestan no sentirse pertenecientes sí participan de una asociación u organización indígena, como se puede observar en la siguiente Tabla 6 .

Respecto del tipo de organización o asociación en que participan aquellos estudiantes que reportaron ser miembros de alguna o varias, tenemos que 5.862 indicaron no pertenecer a alguna o algunas asociaciones u organización indígena, 91 estudiantes declararon pertenecer a un grupo juvenil cultural o estudiantil indígena, 174 estudiantes a cofradías o bailes religiosos, 41 estudiantes a un círculo de hijos de pueblos, 11 estudiantes a una organización política indígena, 141 estudiantes a una asociación indígena, 109 estudiantes a una comunidad indígena, 93 estudiantes a una asociación deportiva indígena, y a otros 263 estudiantes. Los datos son consistentes con la realidad de la Región de Arica y Parinacota y Región de Tarapacá, donde durante el año se evidencia que un número importante de personas participa mediante asociaciones de bailes religiosos, en la Fiesta de las Peñas en Arica y en la fiesta de La Tirana en Iquique.

La información expuesta en la Tabla 7 da cuenta de que en mayor proporción los estudiantes que manifiestan sentirse pertenecientes a un pueblo originario poseen como beneficio el Fondo Solidario de Crédito Universitario, otorgado a la población de menores ingresos del país, lo que da cuenta que una cantidad importante de estudiantes con autoidentificación étnica pertenece al $80 \%$ de 
Tabla 6. Participación en organización o institución étnica.

\begin{tabular}{lccc}
\hline \multirow{2}{*}{$\begin{array}{l}\text { Participa o es miembro de una asociación } \\
\text { u organización indígena? }\end{array}$} & ¿Se siente perteneciente a algún pueblo indígena? & \multirow{2}{*}{ Total } \\
\cline { 2 - 3 } Sí & Sí & No & 1.149 \\
No & 1.044 & 4.327 & 5.862 \\
\hline
\end{tabular}

Tabla 7. Beneficios asociados

¿Posee alguno de los siguientes beneficios?* ¿Se siente perteneciente a algún pueblo originario?

(Tabulación cruzada).

\begin{tabular}{|c|c|c|c|c|}
\hline & \multirow[t]{2}{*}{ Recuento } & \multicolumn{2}{|c|}{$\begin{array}{l}\text { ¿Se siente perteneciente a } \\
\text { algún pueblo originario? }\end{array}$} & \multirow[t]{2}{*}{ Total } \\
\hline & & Sí & No & \\
\hline \multirow{9}{*}{$\begin{array}{l}\text { ¿Posee alguno de } \\
\text { los siguientes beneficios? }\end{array}$} & No tengo beneficios & 545 & 1187 & 1732 \\
\hline & Beca indígena & 204 & 5 & 209 \\
\hline & Beca JUNAEB & 263 & 414 & 677 \\
\hline & Fondo Solidario de Crédito Universitario & 408 & 685 & 1093 \\
\hline & Crédito con Aval del Estado & 148 & 420 & 568 \\
\hline & Beca profesionales de la educación & 8 & 15 & 23 \\
\hline & Beca Funcionarios & 21 & 46 & 67 \\
\hline & Beca Institucional & 14 & 23 & 37 \\
\hline & Otros & 211 & 510 & 721 \\
\hline
\end{tabular}

la población con menores ingresos. En segundo lugar se encuentra la beca alimenticia JUNAEB y, finalmente, la beca indígena, la que además de su requisito académico exige que el beneficiario se encuentre acreditado por CONADI, y que como se expuso con anterioridad, no todos los estudiantes que reportan pertenecer a un pueblo, poseen.

\section{Desafíos en la inclusión en IES de estudiantes de pueblos originarios}

De acuerdo con los datos institucionales acerca de adscripción étnica presentados, es importante tomar en cuenta la variable étnica y su incorporación en la armonización curricular, introduciendo asignaturas y electivos respecto de la atención a la diversidad, porque es importante considerar que la reflexión en la relación entre la adscripción étnica de nuestros estudiantes podría facilitar la promoción y acrecentamiento de la identidad cultural étnica y permitir un diálogo intercultural, donde los estudiantes se puedan articular efectivamente con la lógica educacional oficial y el mundo global actual, pero imbricándose con las características de la identidad cultural regional.
El fortalecer a los estudiantes de los pueblos originarios con un buen diagnóstico y acompañamiento en la universidad, permitiría la existencia de un sujeto agencial, pues la especificidad en nuestro caso de estudio, resulta útil indagar la diferencia basada en la identidad y etnicidad de los contextos locales, pues muchas veces estos no son tan disímiles entre sí, como por ejemplo, en nuestra región que está unida por lazos históricos con pueblos originarios del área centro sur andina existiendo convergencias culturales y sociales entre la población migrante de Perú, Bolivia y Colombia y la población local que potencialmente favorecerían el desarrollo del diálogo intercultural e inclusivo en nuestras aulas (Mondaca y Gajardo, 2013).

Bajo esta consideración, se comprendería por qué la identidad cultural étnica es importante dentro del conjunto de fuentes tan complejas como la nacionalidad, la etnicidad, la clase social, la comunidad, la sexualidad y el género. De allí la necesidad de tratar de alcanzar un acuerdo intercultural donde la identidad étnica se encuentra siempre en formación y evolución constante, en una interacción de negociación cultural que tiene forma de diálogo cultural. 
Las estrategias en materia de Proceso de Enseñanza Aprendizaje de las IES deberían tener una perspectiva de educación inclusiva que considere el diálogo intercultural como un eje central de desarrollo, donde la formación educativa sea una práctica pedagógica que deba considerar: el reconocimiento de la diversidad sociocultural, la participación en la gestión escolar y curricular, la toma de conciencia y la reflexión crítica acerca de lo propio y lo ajeno, la articulación curricular de conocimientos de diferentes universos culturales y la satisfacción de las necesidades básicas del aprendizaje (Mondaca y Gajardo, 2015). Con ello, debería contribuir al mejoramiento de la calidad de vida de los actores socioeducacionales, impulsando relaciones de tolerancia y respeto.

Esta peculiar situación de la región de Arica y Parinacota ubica a nuestra casa de estudios dentro de un escenario transfronterizo que requiere contar con un estudio pormenorizado de las adscripciones y de diversidad cultural presente en sus aulas. Pero el principal problema ante este desafío es que como universidad tenemos que preguntarnos: ¿tenemos las competencias para implementar estrategias de inclusión e interculturalidad en carreras tremendamente marcadas por lo disciplinario?, tal vez a nivel de hipótesis incipiente, es que debemos partir por soluciones simples, como promover electivos de formación general que propicien prácticas pedagógicas y estrategias didácticas de una educación intercultural que pueda articularse efectivamente con la lógica educacional oficial y el mundo global actual, pero imbricándose con las características de la identidad cultural regional.

El concepto de "intercultural" es clave para lograr este objetivo. Haciendo eco de esta propuesta, se puede analizar la ciudadanía más allá de la hegemonía y homogeneidad que ejerce el proceso de globalización (Mondaca, et al., 2014). En este punto, y considerando la especificidad de nuestro caso de estudio, resulta útil indagar la diferencia basada en la identidad y etnicidad de los contextos locales, pues muchas veces estos no son tan disímiles entre sí, como por ejemplo en nuestra región, que está unida por lazos históricos con el área centro sur andina y donde existen convergencias culturales y sociales entre la población migrante y la población local que potencialmente favorecerían el desarrollo del diálogo intercultural e inclusivo en nuestras aulas (Mondaca. et al., 2014b; Winkler y Cueto, 2004).

Desde el ámbito pedagógico y educativo es posible realizar estrategias didácticas que consideren a los migrantes, pueblos originarios y chilenos como parte de una comunidad y de sus instituciones políticas, económicas, sociales y culturales; en una relación de igualdad con sus pares mediante el ejercicio de sus derechos y deberes, donde el Estado-nación es un ente político inclusivo y no un asimilador de diferencias culturales (Mondaca, et al., 2015). En su seno educativo se deberían fomentar los cruces interculturales por razones económicas, comunicativas, cognoscitivas u otras; pero a la vez se deberían acrecentar las posibilidades de diálogo en estos encuentros, reconociendo diferencias y derechos. Lo interesante es que por más que sea una opción que pueda ser criticada, resulta una solución más practicable que la de insistir en una visión tradicional de una ciudadanía asimiladora y homogeneizadora.

Es esta concepción de interculturalidad la que nos permitiría abogar por la inclusión de la diversidad presente en nuestra región y en el Estado nacional, una población comúnmente excluida en la historia del norte de Chile, en el marco de una de las instituciones más importantes de la sociedad: la universidad. Esta propuesta de electivo debe incorporar el fenómeno de la inclusión de migrantes, pueblos originarios y chilenos en las escuelas de la región, debe tomar en consideración a la diversidad desde lineamientos que fomenten la formación desde las aulas de un ciudadano intercultural inclusivo. Para ello, debemos fomentar el desarrollo de habilidades y competencias de inclusión e interculturalidad en los estudiantes (Gimeno, 2008; Martínez, 2008), basadas en el conocimiento de su historia e identidad cultural a nivel local y global. 


\section{Referencias Citadas}

Alvarado, V., Báez, M., Cárcamo, A., Cazenave, M., Del Valle, R., Espinoza, E., García, A., Herrera, R., Lagos, J., Mella, E. y Riquelme, $P$.

2010 "Diversidad y equidad: Programas de acción afirmativa en la educación superior chilena". En: Diagnóstico y Diseño de Intervenciones en Equidad Universitaria. Centro Interuniversitario de Desarrollo CINDA Colección Gestión Universitaria, Santiago.

CASEN

2013 “Grupos de Población: Inmigrantes; Pueblos Indígenas; Adultos Mayores; Género; Niños, Niñas y Adolescentes; Personas con Dificultades y/o Condiciones Permanentes y de Larga Duración". Disponible en: http://www. ministeriodesarrollosocial.gob.cl/resultados-encuestacasen-2013/

Gavilán Vega, Vivian y Lagos Candia, Felipe

¿Integración Social y Subordinación Política de los Aymara?: Un Estudio de la Certificación Estatal Indígena en el Norte Chileno. Estud. atacam. [online]. 2014, n.49 [citado 2016-1118], pp.95-110. Disponible en: <http://www.scielo.cl/scielo. php?script=sci_arttext\&pid=S0718-10432014000300006\&ln $\mathrm{g}=\mathrm{es} \& \mathrm{nrm}=$ iso $>$. ISSN 0718-1043. http://dx.doi.org/10.4067/ S0718-10432014000300006.

Gimeno, $\mathrm{S}$.

2008 Educar por competencias, ¿qué hay de nuevo? , Ediciones Morata, S.L. Madrid

Gundermann, H. y Vergara, J.

2009 Comunidad, organización y complejidad social andinas en el norte de Chile. Estud. atacam. [online]. 2009, n.38 [citado 2016-11-18], pp.107-126. Disponible en: <http:// www.scielo.cl/scielo.php?script=sci_arttext\&pid=S0718$10432009000200008 \& \operatorname{lng}=\mathrm{es} \& n r m=\mathrm{iso}>$. ISSN $0718-1043$. INE http://dx.doi.org/10.4067/S0718-10432009000200008.

2013 Primera Encuesta de Caracterización de la Población Afrodescendiente. Región de Arica y Parinacota. En: http://www.ine.cl/canales/chile_estadistico/ estadisticas_sociales_culturales/etnias/pdf/informe_de_resultados_encuesta_de_caracterizacion_de_la_poblacion_afrodescendiente_de_la_region_de_arica_y_parinacota_2013. pdf

Martínez, J.

2008 "La Ciudadanía se convierte en competencias: Avances y retrocesos". En "Educar por competencias, ¿qué hay de nuevo?". Ediciones Morata, S.L. Madrid.

Ministerio de Educación

2016 LEY NÚM. 20.845. De inclusión escolar que regula la admisión de los y las estudiantes, elimina el financiamiento compartido y prohíbe el lucro en establecimientos educacionales que reciben aportes del Estado. En: https:// www.leychile.cl/Navegar?idNorma=1078172

Ministerio de Desarrollo Social

2013 CASEN 2013. Pueblos Indígenas. Síntesis de Resultados En: http://www.ministeriodesarrollosocial.gob.cl/grupos_poblacion.php

Mondaca, C. y Gajardo, Y.

2013 "La Educación Intercultural Bilingüe en la Región de Arica y Parinacota, 1980-2010". Revista Dialogo Andino. ISSN: ISSN 0719-2681/ISS. Año: 2013.
Vol.: $\mathrm{N}^{\circ}$ 42. Páginas: 68-87. http://www.scielo.c1/scielo. php?pid=S0719-26812013000200007\&script=sci_arttext

Mondaca, C.; Gajardo, Y. y Sánchez, E.

2014 "Historia, memoria y ciudadanía intercultural. El reto del siglo XXI para las aulas de la región de Arica y Parinacota, norte de Chile”. Revista Interciencia, Vol. 39 Nº7. Páginas 524-530. http://www.interciencia.org/v39_07/index.html

Mondaca, C.; Rivera, P. y Gajardo, Y.

2014b "Educación parvularia e inclusión en el norte de Chile. Formando pequeños chilenos en las aulas de Tarapacá". Revista Alpha No 39 Diciembre. Páginas 251-266. http:// www.scielo.cl/pdf/alpha/n39/art_17.pdf

Mondaca, C; Gajardo, Y.; Muñoz, W.; Sánchez, E. Robledo, P. 2015 Estudiantes migrantes en la región de Arica y Parinacota. Caracterización, distribución y consideraciones generales. En: Las fronteras del transnacionalismo. Limites y desbordes de la experiencia migrante en el centro y norte de Chile. Guizardi, M. (Editora). Editorial Ocho Libros Santiago.

Mondaca, C.; Gajardo, Y.

"Interculturalidad, Migrantes y Educación". En Revista "Diálogo Andino" [online]. 2015, n.47, pp. 3-6. ISSN 0719-2681.

Oyarce, A.M., Pedrero M.M y Pérez, G.

2005 "Una investigación operacional sobre criterios de adscripción étnica/ Cultural desde/ sobre ocho pueblos indígenas de Chile: Contribución a los instrumentos de recolección de datos". En: http://www.cepal.org/celade/ noticias/paginas/7/21237/AMOyarce.pdf

UNESCO

1990 Declaración Mundial sobre educación para todos y marco de acción para satisfacer las necesidades básicas de aprendizaje. Jomtien, Tailandia (5 al 9 de marzo de 1990). En: http://www.unesco.org/education/pdf/JOMTIE_S.PDF UNESCO

2015 La Educación para Todos, 2000-2015: logros y desafíos. Organización de las Naciones Unidas para la Educación, la Ciencia y la Cultura 7, París, Francia. En: http://unesdoc. unesco.org/images/0023/002325/232565s.pdf

UNESCO

2015 Declaración de Incheon. Educación 2030. Hacia una educación inclusiva y equitativa de calidad y un aprendizaje a lo largo de la vida para todos. Páginas 31-41. En: http:// unesdoc.unesco.org/images/0023/002338/233813M.pdf

Universidad de Tarapacá

2012 “Sistema de Dirección Estratégica. 2011-2016". En: http://www.uta.cl/UTAALDIA/acreditacion/planestrategico2011_2016.pdf

Universidad de Tarapacá

2012 Modelo Educativo de la Universidad de Tarapacá. En: http://cidduta.uta.cl/download/modelo_educativo.pdf

UTA MINEDUC

2015 Proyecto: "Generación de valor a través de la innovación curricular y del mejoramiento de la calidad de los grados y títulos ofrecidos por la Universidad de Tarapacá". Convenio de Desempeño de Armonización Curriuclar UTA 1501. Ministerio de Educación. Chile.

UTA MINEDUC

2013 Proyecto "Educación de calidad para tod@s: un compromiso con la formación inicial de profesores de la 
Universidad de Tarapacá" Convenio de Desempeño de Formación Inicial de Profesores UTA 1309. Ministerio de Educación. Chile.
Winler, D. y Cueto, S.

2004 Etnicidad, Raza, Género y Educación en América Latina. Ed. PREAL.

\section{Notas}

1 Programa de "Educación para Todos" (EPT) en Jomtien (ratificado en Dakar el año 2000).

2 Aun pese a no haberse alcanzado las metas propuestas, el 2014 en el Acuerdo de Mascate, y con la labor desempeñada con el Grupo de Trabajo Abierto acerca de los Objetivos de Desarrollo Sostenible, se reconoce la contribución de la Iniciativa Mundial "La educación ante todo", para impulsar el compromiso político en pro de una educación más inclusiva.

3 Una nueva visión de la educación que propone: "Garantizar una educación inclusiva, equitativa y de calidad y promover oportunidades de aprendizaje durante toda la vida para todos" (Foro Mundial sobre la Educación 2015), convirtiéndose en una perspectiva de futuro transformadora en temas exclusión y marginación (igualdad de género, calidad de la educación, oportunidades de aprendizaje a lo largo de la vida, etc.).

4 "Garantizar una educación inclusiva, equitativa y de calidad y promover oportunidades de aprendizaje durante toda la vida para todos”. Buscando: “... lograr la igualdad entre los géneros y el empoderamiento de todas las mujeres y niñas.”, como también, “...Promover sociedades pacíficas e inclusivas para el desarrollo sostenible, facilitar el acceso a la justicia para todos y crear instituciones eficaces, responsables e inclusivas a todos los niveles" (UNESCO, 2015:330-331).

5 Todo esto, relacionado con las metas propuestas por la UNESCO (2015: 334, 336) de que para el 2030, a lo menos se debería: “.... asegurar el acceso en condiciones de igualdad para todos los hombres y las mujeres a formación técnica, profesional y superior asequible y de calidad, incluida la enseñanza universitaria.”, y también se deberían: “...eliminar las disparidades de género en la educación y garantizar el acceso en condiciones de igualdad de las personas vulnerables, incluidas las personas con discapacidad, los pueblos indígenas y los niños en situaciones de vulnerabilidad, a todos los niveles de la enseñanza y la formación profesional".

$6 \quad$ Esto es visible hasta hoy en diversas manifestaciones culturales de la población regional, donde lo indígena, lo afro y lo español están potentemente imbricados.

7 Una iniciativa desde los propios estudiantes surgió a fines de la década del noventa (1999), al crearse bajo el alero de la universidad la Asociación de Estudiantes de Pueblos Originarios (AESPO) que mantiene hasta hoy un trabajo con los estudiantes pertenecientes a los pueblos originarios. Incluso manejando hoy una residencia universitaria indígena.
8 También es importante señalar que se implementó en dos etapas (2006-2009-2012) un programa de acción afirmativa denominado Takhi (camino en aymara), financiado por la Fundación Ford que tenía como propósito:

"Evaluar y promover políticas e iniciativas de acción afirmativa en beneficio de la comunidad universitaria perteneciente a la cultura aimara u otro pueblo originario, que permitan incrementar significativamente sus indicadores de desempeño académico en las carreras de pregrado favoreciendo de esta manera su incorporación a las carreras de postgrado" (Alvarado, et al., 2010:205). Este programa fundamentalmente desarrolló programas de tutorías y cursos de reforzamiento en las áreas más deficitarias de los alumnos con raigambre indígena y tenía como objetivos de la segunda etapa: "Consolidar e incorporar un conjunto de acciones afirmativas en la Universidad de Tarapacá para estudiantes indígenas, transformándolas en políticas institucionales que favorezcan la inclusión en el contexto universitario. Promover la integración universitaria en la Macro Región Centro Sur Andina, apoyando la Incorporación de acciones afirmativas, a partir de la experiencia adquirida por el Programa Thakhi. Disponible en: http://nebula.wsimg.com/7f9fab443f5b8eb7d3c3f7cb 3c2f73f5?AccessKeyId=0D302DDF09BBFDD7F719\&d isposition $=0$ \&alloworigin $=1$

9 Una oportunidad interesante de hacer notar es que este año 2016 la universidad comenzó un proceso de armonización curricular (Convenio de Desempeño UTA-MINEDUC 1501), lo que podría permitir integrar la variable de inclusión étnica en todo su currículum. Otro gran desafío en materia de inclusión es definir una política institucional para trabajar las Necesidades Educativas Específicas o con capacidades diferentes. Es importante señalar que en la actualidad se desenvuelven en los diferentes campus de la Universidad aproximadamente 16 alumnos con discapacidades visibles, desconociéndose aquellos que poseen discapacidades no visibles. La SEREMIA de Educación de la Región de Arica y Parinacota informó el 2015 de un potencial de 66 alumnos con discapacidades no visibles diagnosticadas cursando tercero y cuarto año medio, respectivamente, entre estos sus diagnósticos son: dificultades específicas del aprendizaje; discapacidad intelectual leve; discapacidad intelectual moderada; discapacidad motora grave; discapacidad motora leve; discapacidad motora moderada; graves alteraciones de la relación y de la comunicación; hipoacusia severa; coeficiente intelectual en rango limítrofe; trastorno de déficit atencional; trastorno del espectro autista, entre otros. 

ANDROS IMPRESORES

www.androsimpresores.cl 